\title{
DNA Relatedness among the Pathovar Strains of Pseudomonas syringae subsp. savastanoi Janse (1982) and Proposal of Pseudomonas savastanoi sp. nov.
}

\author{
L. GARDAN, ${ }^{1 *}$ C. BOLLET, ${ }^{2}$ M. ABU GHORRAH, ${ }^{1} \dagger$ F. GRIMONT, ${ }^{3}$ AND P. A. D. GRIMONT ${ }^{3}$ \\ Institut National de la Recherche Agronomique, Station de Pathologie Végétale, F-49070 Beaucouzé, ${ }^{1}$ \\ Laboratoire de Bactériologie et d'Hygiène Hospitalière, Hôpital Salvator, F-13009 Marseille, ${ }^{2}$ \\ and Unité des Entérobactéries, Institut National de la Santé et de la Recherche \\ Médicale U199, Institut Pasteur, F-75724 Paris Cedex $15,{ }^{3}$ France
}

\begin{abstract}
We found that Pseudomonas syringae subsp. savastanoi strains belong to a DNA relatedness group that includes strains of $P$. syringae pv. glycinea and $P$. syringae pv. phaseolicola. This DNA group was distinct from $P$. syringae pv. syringae (including the type strain of $P$. syringae). The results of a numerical analysis were in accord with DNA hybridization data. Thus, $P$. syringae subsp. savastanoi (Janse) 1982 is elevated to species level as Pseudomonas savastanoi sp. nov., which includes $P$. savastanoi pv. savastanoi, $P$. savastanoi pv. glycinea, and $P$. savastanoi pv. phaseolicola.
\end{abstract}

In 1908, Smith (22) named "Bacterium savastanoi," the bacterium which causes knots on several plants belonging to the family Oleaceae. This species was later transferred to the genus Pseudomonas as "Pseudomonas savastanoi" by Stevens (24).

In 1978, Young et al. (27) proposed a new nomenclature and classification for plant-pathogenic bacteria and introduced the concept of pathovar, and all fluorescent oxidasenegative Pseudomonas species (except Pseudomonas viridiflava) were considered to be members of a single species, Pseudomonas syringae, which had a number of pathovars. Thus, " $P$. savastanoi" became $P$. syringae pv. savastanoi (27). The pathovar is an infrasubspecific subdivision which is not covered by the International Code of Nomenclature of Bacteria (14). Thus, the name $P$. syringae pv. savastanoi did not appear on the Approved Lists of Bacterial Names (21).

"Bacterium savastanoi var. fraxini"' (2), which was isolated from excrescences on Fraxinus excelsior L., and "Bacterium tonellianum" (6), which was isolated by Ferraris from galls on Nerium oleander $\mathrm{L}$. and which became " $P$. savastanoi var. nerii"' (21), were included in $P$. syringae pv. savastanoi by Young et al. (27).

In 1982, Janse revived the epithet savastanoi in designating a new subspecies, Pseudomonas syringae subsp. savastanoi (17). The following three pathovars were recognized in this subspecies: $P$. syringae subsp. savastanoi pv. oleae, which causes parenchymatic galls on members of various genera of the Oleaceae; $P$. syringae subsp. savastanoi pv. nerii, which causes parenchymatic galls or wartlike excrescences on $N$. oleander $\mathrm{L}$. and members of various genera of the Oleaceae; and $P$. syringae subsp. savastanoi pv. fraxini, which causes wartlike excrescences on $F$. excelsior $L$. and Olea europea L. (12). Although Janse did not specifically describe the taxon, his proposal in fact created $P$. syringae subsp. syringae (14).

Several authors have described physiological, nutritional,

\footnotetext{
* Corresponding author.

† Present address: Department of Plant Protection, Faculty of Agronomy, University of Damas, Damas, Syria.
}

and biochemical characteristics of $P$. syringae subsp. savastanoi strains isolated from different hosts and compared these organisms with some pathovars of $P$. syringae subsp. syringae $(10,11,16,25)$.

In a numerical taxonomy study, 34 strains of $P$. syringae subsp. savastanoi constituted a phenon that was closely related to $P$. syringae pv. glycinea and $P$. syringae pv. phaseolicola (20).

Previously, DNA-DNA hybridization data have shown that $P$. syringae is a heterogeneous species (18).

The purpose of this work was to determine the taxonomic position of $P$. syringae subsp. savastanoi and related pathovars by using numerical taxonomy and DNA-DNA hybridization. Our results indicated that $P$. syringae subsp. savastanoi should be elevated to species level as Pseudomonas savastanoi sp. nov.

\section{MATERIALS AND METHODS}

Bacterial strains. Three sets of strains were used. The first set comprised $143 P$. syringae subsp. savastanoi strains that were isolated from various hosts (Table 1$)$. The second set included 50 reference strains of $P$. syringae pathovars and the type strains of Pseudomonas cichorii, $P$. viridiflava, and Pseudomonas amygdali; the origins of these strains have been published previously (7). The third set contained 35 strains of miscellaneous Pseudomonas spp. (Table 2), Agrobacterium tumefaciens CFBP $2413^{\mathrm{T}}$ ( $\mathrm{T}=$ type strain), and Xanthomonas campestris pv. campestris CFBP 2350. All of the bacteria were cultured routinely on YBGA (yeast extract, $7 \mathrm{~g}$; Bacto Peptone, $7 \mathrm{~g}$; glucose, $7 \mathrm{~g}$; agar, $15 \mathrm{~g}$; distilled water, $1,000 \mathrm{ml} ; \mathrm{pH} 7.2$ ) incubated at $25^{\circ} \mathrm{C}$.

Biochemical and physiological tests. The presence of oxidase, gelatinase, and arginine dihydrolase, fluorescent pigment production, levan formation, acid production from sucrose, sorbitol, erythritol, and mannitol, reduction of nitrate, hydrolysis of Tween 80 and esculin, and the hypersensitivity reaction on tobacco leaves were tested as described by Lelliott et al. (15). Pectolytic activity was tested by using the method of Prunier and Kaiser (19) and on 
TABLE 1. Origins of the $143 P$. syringae subsp. savastanoi strains tested

\begin{tabular}{|c|c|c|}
\hline $\begin{array}{l}\text { No. of } \\
\text { strains }\end{array}$ & Host & $\begin{array}{l}\text { Geographic origin } \\
\text { (no. of strains) }\end{array}$ \\
\hline 58 & Olive & $\begin{array}{l}\text { Algeria (31), Tunisia (1), } \\
\text { France (7), Portugal (1), } \\
\text { United States (2), Yugoslavia } \\
\text { (4), Greece (1), Italy (7), } \\
\text { Syria (4) }\end{array}$ \\
\hline 33 & Oleander & $\begin{array}{l}\text { France (13), Algeria (4), Greece } \\
\text { (5), Yugoslavia (1), } \\
\text { Netherlands (1), United } \\
\text { States (4), Italy (5) }\end{array}$ \\
\hline 39 & Ash & $\begin{array}{l}\text { France (25), Algeria (2), United } \\
\text { Kingdom (2), United States } \\
\text { (1), Netherlands (9) }\end{array}$ \\
\hline 6 & Privet & Italy (6) \\
\hline 4 & Phillyrea sp. & Algeria (4) \\
\hline 3 & Jasmine & Greece (3) \\
\hline
\end{tabular}

pectate gels at $\mathrm{pH} 5$ and 8 (9). Utilization of DL-lactate, $\mathrm{L}-(+)$-tartrate, and $\mathrm{D}-(-)$-tartrate was tested on a basal medium $\left(\mathrm{NH}_{4} \mathrm{H}_{2} \mathrm{PO}_{4}, 1 \mathrm{~g} ; \mathrm{KCl}, 0.2 \mathrm{~g} ; \mathrm{MgSO}_{4} \cdot 2 \mathrm{H}_{2} \mathrm{O}, 0.2 \mathrm{~g}\right.$; agar, $3 \mathrm{~g}$; bromothymol blue, $0.08 \mathrm{~g}$; distilled water, 1,000 $\mathrm{ml} ; \mathrm{pH}$ 7.2) supplemented with $0.1 \%$ (wt/vol) organic acid (sodium salt). The presence of DNase was tested on DNA agar (Diagnostics Pasteur, Marnes-la-Coquette, France).

Assimilation of 49 carbohydrates, 49 organic acids, and 49 amino acids was studied by using API 50CH, API 50AO, and API 50AA strips (BioMérieux, La Balme-les-Grottes, France) which were incubated at $24^{\circ} \mathrm{C}$ and examined for growth after 6 days.

Numerical analysis. A total of 167 characters were included in the numerical taxonomy analysis. The distance matrix was calculated by using the Jaccard coefficient (23). Cluster analysis was done by using the unweighted pair group method with averages (23).

DNA extraction. Previously described methods were used to extract and purify DNA (1).

DNA-DNA hybridization. Native DNAs were labeled in vitro by nick translation with tritium-labeled nucleotides (Amersham International, Amersham, England). The procedure used for the hybridization experiments (S1 nucleasetrichloroacetic acid method) has been described previously $(3,8)$. The reassociation temperature was $60^{\circ} \mathrm{C}$. DNA-DNA hybridization tests were carried out by using labeled DNAs from $P$. syringae pv. syringae CFBP $1392^{\mathrm{T}}$ and $P$. syringae subsp. savastanoi CFBP $1670^{\mathrm{T}}$.

Thermal stability of reassociated DNAs. The temperature at which $50 \%$ of reassociated DNA became hydrolyzable by the $\mathrm{S} 1$ enzyme $\left(T_{m}\right)$ was determined by using the method of

TABLE 2. Origins of 33 fluorescent and nonfluorescent oxidase-positive Pseudomonas strains and $A$. tumefaciens and $X$. campestris pv. campestris strains

\begin{tabular}{|c|c|c|}
\hline Taxon & CFBP no. & Host or origin and place and year of isolation \\
\hline $\begin{array}{l}\text { Pseudomonas aeruginosa } \\
P . \text { alcaligenes } \\
P . \text { caryophylli } \\
P . \text { cepacia } \\
P . \text { cepacia } \\
P . \text { corrugata } \\
P . \text { corrugata } \\
P . \text { corrugata } \\
P . \text { corrugata } \\
P . \text { fluorescens } \\
P . \text { fluorescens } \\
P . \text { fluorescens } \\
P . \text { fluorescens } \\
P . \text { fluorescens } \\
P . \text { fluorescens } \\
P . \text { fluorescens } \\
P . \text { fuscovaginae } \\
P . \text { gladioli pv. gladioli } \\
P . \text { gladioli pv. alliicola } \\
P . \text { marginalis pv. marginalis } \\
P . \text { marginalis pv. pastinacae } \\
P . \text { marginalis pv. alfalfae } \\
P . \text { putida } \\
P . \text { putida } \\
P . \text { pseudoalcalignenes } \\
P . \text { rubrilineans } \\
P . \text { rubrisubalbicans } \\
P . \text { solanacearum } \\
P . \text { solanacearum (race } 3 \text { ) } \\
P . \text { solanacearum (race } 2) \\
P . \text { stutzeri } \\
P . \text { tolaasii } \\
P . \text { tolaasii } \\
\text { A. tumefaciens } \\
X . \text { campestris pv. campestris }\end{array}$ & $\begin{array}{l}2466^{\mathrm{T}} \\
2437^{\mathrm{T}} \\
2429^{\mathrm{T}} \\
2227^{\mathrm{T}} \\
2234 \\
2431^{\mathrm{T}} \\
145.41 \\
30.5 \\
22.4 \\
2102^{\mathrm{T}} \\
2123 \\
2125 \\
2127 \\
2129 \\
2130 \\
2299 \\
2065^{\mathrm{T}} \\
2427^{\mathrm{T}} \\
2422 \\
1387^{\mathrm{T}} \\
2038^{\mathrm{T}} \\
2039^{\mathrm{T}} \\
2066^{\mathrm{T}} \\
2298 \\
2435^{\mathrm{T}} \\
1294 \\
1296 \\
2047^{\mathrm{T}} \\
1420 \\
1482 \\
2443^{\mathrm{T}} \\
2068^{\mathrm{T}} \\
2152 \\
2413^{\mathrm{T}} \\
2350\end{array}$ & $\begin{array}{l}\text { Host, origin, and year unknown } \\
\text { Water, origin and year unknown } \\
\text { Dianthus caryophyllus, United States, } 1951 \\
\text { Allium cepa, United States, year unknown } \\
\text { Hospital, France, year unknown } \\
\text { Lycopersicum esculentum, United Kingdom, } 1972 \\
\text { Lycopersicon esculentum, France, } 1982 \\
\text { Lycopersicon esculentum, France, 1984 } \\
\text { Lycopersicon esculentum, France, 1983 } \\
\text { Water, United Kingdom, 1951 } \\
\text { Water, Netherlands, 1966 } \\
\text { Host, origin, and year unknown } \\
\text { Egg, United States, year unknown } \\
\text { Water, origin and year unknown } \\
\text { Water, origin and year unknown } \\
\text { Beta vulgaris (root), France, 1983 } \\
\text { Oryza sativa, Japan, 1976 } \\
\text { Gladiolus sp., origin unknown, 1966 } \\
\text { Allium cepa, United States, 1939 } \\
\text { Cichorium intybus, United States, } 1949 \\
\text { Pastinaca sativa, United States, } 1959 \\
\text { Medicago sativa, United States, } 1971 \\
\text { Soil, United States, year unknown } \\
\text { Malus sylvestris (root), France, } 1983 \\
\text { Sinus drainage, origin unknown, 1966 } \\
\text { Saccharum officinale, Réunion, } 1970 \\
\text { Saccharum officinale, Réunion, } 1970 \\
\text { Lycopersicon esculentum, United States, } 1953 \\
\text { Solanum phureja, Colombia, 1965 } \\
\text { Musa sp., Panama, 1958 } \\
\text { Spinal fluid, United States, 1970 } \\
\text { Agaricus bisporus, United Kingdom, } 1965 \\
\text { Agaricus bisporus, France, 1975 } \\
\text { Host plant and origin unknown, 1972 } \\
\text { Brassica oleracea, United Kingdom, 1957 }\end{array}$ \\
\hline
\end{tabular}




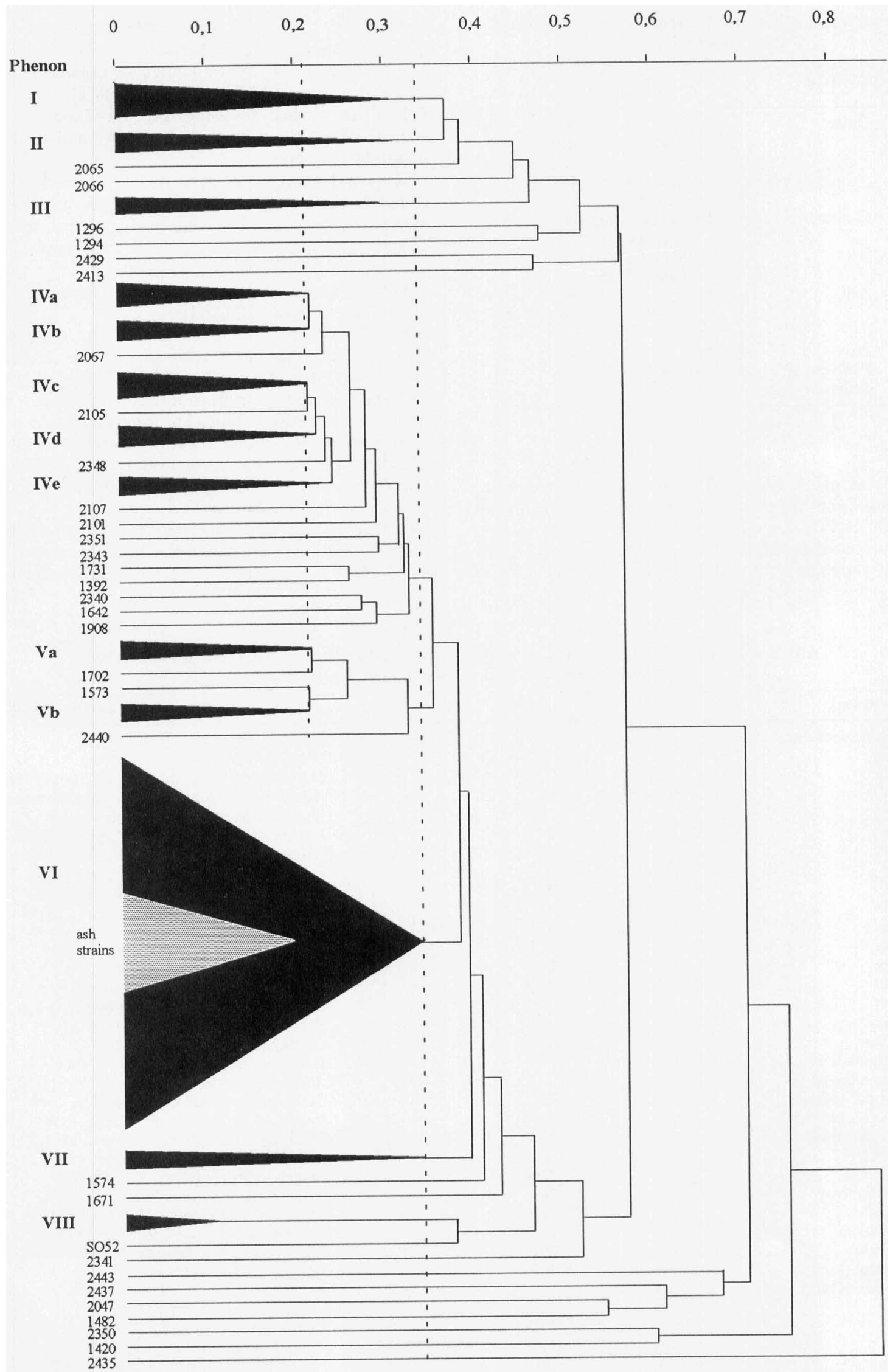

FIG. 1. Dendrogram of the distances among 231 strains of $P$. savastanoi, $P$. syringae sensu lato, and related Pseudomonas spp. 
TABLE 3. Distribution of species and pathovars among eight phena

\begin{tabular}{|c|c|c|c|}
\hline Phenon or group & Subphenon & $\begin{array}{l}\text { No. of } \\
\text { strains }\end{array}$ & Strain(s) or no. of strains \\
\hline I & & 15 & $\begin{array}{l}P . \text { cornugata CFBP } 2431^{\mathrm{T}} \text {, CFBP } 145.41, \text { CFBP } 30.5 \text {, and CFBP } 22.4, P \text {. tolaasii } \\
\text { CFBP } 2068^{\mathrm{T}} \text { and CFBP } 2152, P \text {. fluorescens CFBP } 2299 \text {, CFBP } 2129 \text {, CFBP } \\
2102^{\mathrm{T}} \text {, CFBP } 2123 \text {, and CFBP } 2125, P \text {. marginalis pv. pastinacea CFBP } 2038^{\mathrm{T}} \text {, } \\
P . \text { marginalis pv. marginalis CFBP } 1387^{\mathrm{T}}, P \text {. marginalis pv. alfalfae CFBP } \\
2039^{\mathrm{T}}, P \text {. putida CFBP } 2298\end{array}$ \\
\hline II & & 3 & $P$. fluorescens CFBP 2127 and CFBP $2130, P$. aeruginosa CFBP $2466^{\mathrm{T}}$ \\
\hline III & & 4 & $\begin{array}{l}\text { P. gladioli pv. gladioli CFBP } 2427^{\mathrm{T}}, P \text {. gladioli pv. alliicola CFBP } 2422, P \text {. cepacia } \\
\text { CFBP } 2227^{\mathrm{T}} \text {, and CFBP } 2234\end{array}$ \\
\hline \multirow[t]{6}{*}{ IV } & IVa & 3 & $\begin{array}{l}\text { P. syringae pv. photiniae CFBP 2899, } P \text {. syringae pv. myricae CFBP 2897, } P \text {. } \\
\text { syringae pv. aesculi CFBP } 2894\end{array}$ \\
\hline & IVb & 2 & $P$. syringae pv. thea CFBP $2353, P$. syringae pv. tagetis CFBP 1694 , \\
\hline & IVc & $1 \overline{1}$ & $\begin{array}{l}P \text { syringae pv. japonica CFBP } 2896, P \text {. syringae pv. hibisci CFBP } 2895, P \text {. } \\
\text { syringae pv. mellea CFBP } 2344, P \text {. syringae pv. tabaci CFBP } 2106, P \text {. syringae } \\
\text { pv. dysoxyli CFBP } 2356, P \text {. syringae pv. aptata CFBP } 1617, P \text {. syringae pv. } \\
\text { panici CFBP } 2345, P \text {. syringae pv. papulans CFBP } 1754, P \text {. syringae pv. } \\
\text { atrofaciens CFBP } 2213, P \text {. syringae pv. primulae CFBP } 1660, P \text {. syringae pv. } \\
\text { aceris CFBP } 2339\end{array}$ \\
\hline & IVd & 3 & $\begin{array}{l}P \text { syringae pv. tomato CFBP } 2212, P \text {. syringae pv. maculicola CFBP } 1657, P \text {. } \\
\text { syringae pv. apii CFBP } 2103\end{array}$ \\
\hline & IVe & 4 & $\begin{array}{l}P \text {. syringae pv. passiflorae CFBP } 2346, P \text {. syringae pv. delphinii CFBP } 2215, P \text {. } \\
\text { syringae pv. coronafaciens CFBP } 2216, P \text {. syringae pv. striafaciens CFBP } 1674\end{array}$ \\
\hline & & 12 & $\begin{array}{l}\text { Isolated phenotypes of } P \text {. syringae pv. helianthi CFBP } 2067, P \text {. syringae pv. pisi } \\
\text { CFBP } 2105, P \text {. syringae pv. ribicola CFBP } 2348 ; P \text {. syringae pv. viridiflava } \\
\text { CFBP 2107, } P \text {. syringae pv. cichorii CFBP } 2101, P \text {. syringae pv. morsprunorum } \\
\text { CFBP 2351, } P \text {. syringae pv. eriobotryae CFBP } 2343, P \text {. syringae pv. lapsa } \\
\text { CFBP } 1731, P \text {. syringae pv. syringae CFBP } 1392, P \text {. syringae pv. atropurpurea } \\
\text { CFBP } 2340, P \text {. syringae pv. mori CFBP } 1642, P \text {. syringae pv. porri CFBP } 1908\end{array}$ \\
\hline \multirow[t]{2}{*}{ V } & $\mathrm{Va}$ & 4 & $\begin{array}{l}P \text {. syringae pv. philadelphia CFBP } 2898, P \text {. syringae pv. berberidis CFBP } 1727, P \text {. } \\
\text { syringae pv. anthrirrhini CFBP } 1620, P \text {. syringae pv. viburni CFBP } 1702\end{array}$ \\
\hline & $\mathrm{Vb}$ & $\begin{array}{l}4 \\
1\end{array}$ & $\begin{array}{l}P \text {. syringae pv. persicae CFBP } 1573 \text {, W } 24-1 \text {, W } 24-2 \text {, and W } 24-3 \\
\text { Isolated phenotype of } P \text {. syringae pv. lacrymans CFBP } 2440\end{array}$ \\
\hline VI & & 142 & $\begin{array}{l}\text { P. syringae subsp. savastanoi ( } 57 \text {, strains), strains from oleander ( } 33 \text { strains), ash } \\
\text { ( } 39 \text { strains), privet ( } 6 \text { strains), Phillyrea sp. ( } 4 \text { strains), and jasmine ( } 3 \text { strains) }\end{array}$ \\
\hline VII & & 3 & $\begin{array}{l}\text { P. syringae pv. glycinea CFBP 2214, } P \text {. syringae pv. phaseolicola CFBP } 1390, P \text {. } \\
\text { syringae pv. ulmi CFBP } 1407\end{array}$ \\
\hline VIII & & 2 & $P$. amygdali CFBP 2354 and CFBP W28-1 \\
\hline Isolated phenotypes & & 17 & 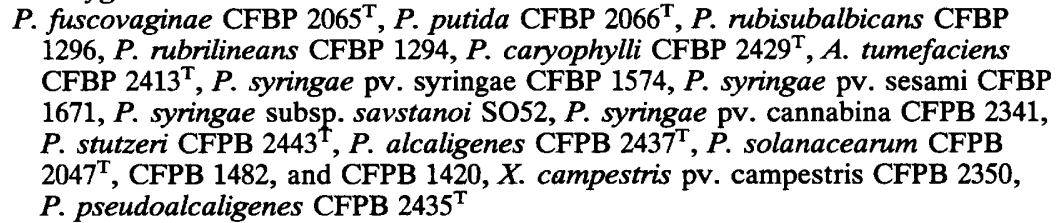 \\
\hline
\end{tabular}

Crosa et al. (3). The $\Delta T_{m}$ was the difference between the $T_{m}$ of the heteroduplex and the $T_{m}$ of the homoduplex.

\section{RESULTS}

Numerical analysis. A dendrogram displaying the distance relationships among the 231 strains which we studied is shown in Fig. 1.

At a distance of 0.346 , eight phena and 17 isolated strains were observed. The distribution of species, subspecies, and pathovars in the eight major phena is shown in Table 3.

Phena I and II contained fluorescent oxidase-positive Pseudomonas strains. Phenon III contained only nonfluorescent phytopathogenic Pseudomonas strains.

Phena IV to VIII included reference strains of fluorescent and nonfluorescent ( $P$. amygdali) phytopathogenic Pseudomonas spp. that were either oxidase negative or oxidase positive $(P$. cichorii), corresponding to groups I through III of Lelliott et al. (15).

Phenon IV was subdivided into five subphena (subphena
IVa to IVe), and phenon $\mathrm{V}$ was subdivided into subphena $\mathrm{Va}$ and $\mathrm{Vb}$.

Phenon VI contained $P$. syringae subsp. savastanoi strains. All but one strain of $P$. syringae subsp. savastanoi fell into this phenon. All 39 strains isolated from ash were clustered in one subphenon. The strains isolated from olive and oleander did not constitute a subphenon within phenon VI.

Only 4 of the 17 unclustered strains were identified as $P$. syringae strains $(P$. syringae subsp. savastanoi SO52, $P$. syringae pv. cannabina CFBP $2341, P$. syringae pv. syringae CFBP 1574, and $P$. syringae pv. sesami CFBP 1671) (Table 3).

Phena I to III were clearly differentiated by biochemical tests from phena IV to VIII. Strains in phena IV to VIII could not utilize $N$-acetylglucosamine, acetate, $N$-valerate, arginine, spermine, tyrosine, 2-ketogluconate, ethanolamine, L-tryptophan, L-ornithine, DL-kynurenine, isobutyrate, citraconate, itaconate, mesaconate, levulinate, and 
TABLE 4. Characteristics that differentiate phena IV to VIII

\begin{tabular}{|c|c|c|c|c|c|c|c|c|c|c|c|c|}
\hline \multirow{2}{*}{$\begin{array}{l}\text { Phenon or } \\
\text { subphenon }\end{array}$} & \multicolumn{10}{|c|}{ Utilization of: } & \multirow{2}{*}{$\begin{array}{c}\text { Levan } \\
\text { production }\end{array}$} & \multirow{2}{*}{$\begin{array}{l}\text { Fluore- } \\
\text { scence }\end{array}$} \\
\hline & $\begin{array}{c}\text { meso- } \\
\text { Tartrate }\end{array}$ & $\begin{array}{l}\text { DL-Hydroxy- } \\
\text { butyrate }\end{array}$ & $\begin{array}{l}\text { Eryth- } \\
\text { ritol }\end{array}$ & DNase & Esculin & $\begin{array}{c}\mathrm{L}-(+)- \\
\text { Tartrate }\end{array}$ & DL-Lactate & Glucosamine & $\beta$-Alanine & $\begin{array}{l}\mathrm{D}-(-)- \\
\text { Tartrate }\end{array}$ & & \\
\hline IVa & $+^{a}$ & - & - & + & - & + & - & - & - & - & + & + \\
\hline $\mathrm{IVb}$ & + & d & - & - & - & d & - & + & + & - & + & + \\
\hline IVc & + & + & + & - & + & d & d & $\mathrm{d}$ & - & d & + & + \\
\hline IVd & + & + & - & d & - & - & + & - & - & + & + & + \\
\hline IVe & + & - & + & - & + & - & - & - & - & - & + & + \\
\hline $\mathrm{Va}$ & + & - & - & d & d & - & - & - & - & + & + & + \\
\hline $\mathrm{Vb}$ & - & d & - & + & - & - & - & - & - & + & + & + \\
\hline VI & - & - & - & + & - & + & - & - & - & - & - & + \\
\hline VII & $d$ & - & d & d & - & + & - & - & - & d & + & + \\
\hline VIII & - & - & - & - & - & - & - & - & - & - & + & - \\
\hline
\end{tabular}

${ }^{a}+, 90$ to $100 \%$ of the strains are positive;,- 0 to $10 \%$ of the strains are positive; $d, 11$ to $89 \%$ of the strains are positive.

$p$-aminobenzoate and could not reduce nitrate. All of the strains in phena I to III gave the opposite reactions.

Characteristics that differentiate phena IV to VIII are shown in Table 4. Identification of a strain as a phenon IV or $\mathrm{V}$ strain requires identification at the subphenon level (Table 4). The reactions exhibited by the phenon VI strains differed somewhat when the source of isolation was considered (Table 5).

DNA relatedness. Results of DNA relatedness experiments are shown in Table 6. $P$. syringae subsp. savastanoi strains isolated from six hosts were 75 to $100 \%$ related to type strain CFBP 1670. For the two lowest relatedness values ( 75 and $79 \%$ ), the $\Delta T_{m}$ values were 1.5 and $3.0^{\circ} \mathrm{C}$, respectively. The reference strains of $P$. syringae pv. glycinea and $P$. syringae pv. phaseolicola were 72 and $83 \%$ related to strain CFBP $1670^{\mathrm{T}}$, respectively. The $\Delta T_{m}$ value calculated for $P$. syringae pv. glycinea and $P$. syringae subsp. savastanoi hybridized DNAs was $2.5^{\circ} \mathrm{C}$. Thus, $P$. syringae subsp. savastanoi, $P$. syringae pv. glycinea, and $P$. syringae pv. phaseolicola are members of a single DNA hybridization group.

The type strain of $P$. syringae pv. syringae, strain CFBP 1392, was $43 \%$ related to strain CFBP $1670^{\mathrm{T}}$ when DNA from strain CFBP $1670^{\mathrm{T}}$ was labeled; the level of relatedness was $54 \%$ when DNA from strain CFBP $1392^{\mathrm{T}}$ was labeled. $P$. syringae pv. persicae, $P$. syringae pv. tomato, and the other Pseudomonas species which we studied were less than $51 \%$ related to either $P$. syringae subsp. savastanoi CFBP $1670^{\mathrm{T}}$ or $P$. syringae pv. syringae CFBP $1392^{\mathrm{T}}$; thus, these strains are members of DNA groups other than the $P$. syringae $\mathrm{pv}$. syringae or $P$. syringae subsp. savastanoi DNA group. In this study the $P$. syringae pv. syringae DNA group was represented only by the type strain.

\section{DISCUSSION}

Before 1978, a Pseudomonas strain that was isolated for the first time from a new diseased host was considered a species. Young et al. (27) and Dye et al. (5) considered the named, phytopathogenic, oxidase-negative fluorescent, Pseudomonas spp. to be pathovars of $P$. syringae. At the present time, 45 pathovars are recognized $(5,26)$.

Janse (11) found that strains of $P$. syringae pv. savastanoi that were isolated from different hosts had nearly identical biochemical and physiological characteristics. Variations were observed only in the production of levan, in the hydrolysis of pectate, and in the production of indoleacetic acid and cytokininlike compounds. Janse also observed a marked variation in the results of pathogenicity tests among the strains that were isolated from different hosts. For these reasons he named this group of strains $P$. syringae subsp. savastanoi and proposed the following three pathovars: $P$. syringae subsp. savastanoi pv. oleae, $P$. syringae subsp. savastanoi pv. nerii, and $P$. syringae subsp. savastanoi pv. fraxini $(12,17)$.

Cross-pathogenicity is currently being tested by one of us (L.G.) to determine whether pathogenicity characteristics match taxonomic grouping. Pathogenicity of olive strains on oleander has not been demonstrated $(6 \mathrm{a}, 11)$. All strains of $P$. syringae subsp. savastanoi are pathogenic on ash. Ash strains are pathogenic only on ash, and $80 \%$ of oleander strains are pathogenic on olive. However, cross-pathogenicity data for different hosts are not complete.

On the basis of DNA-DNA hybridization, physiological and biochemical characteristics, we propose that $P$. syringae subsp. savastanoi should be elevated to species level as

TABLE 5. Reactions of phenon VI strains ( $P$. syringae subsp. savastanoi) isolated from different hosts

\begin{tabular}{|c|c|c|c|c|c|c|c|c|c|c|c|c|c|}
\hline \multirow{3}{*}{ Host } & \multirow{3}{*}{$\begin{array}{l}\text { No. of } \\
\text { isolates }\end{array}$} & \multicolumn{12}{|c|}{$\%$ of strains positive } \\
\hline & & \multicolumn{10}{|c|}{ Utilization of: } & \multirow{2}{*}{$\begin{array}{l}\text { Hydrolysis of } \\
\text { polypectate } \\
\text { (pH 5) }\end{array}$} & \multirow{2}{*}{$\begin{array}{l}\text { Levan } \\
\text { produc- } \\
\text { tion }\end{array}$} \\
\hline & & L-Serine & n-Caproate & $\begin{array}{l}\text { DL-5-Amino- } \\
\text { valerate }\end{array}$ & L-Leucine & Raffinose & $\begin{array}{l}\text { 2-Amino- } \\
\text { benzoate }\end{array}$ & $\begin{array}{l}\text { L-Tyro- } \\
\text { sine }\end{array}$ & D-Xylose & $\begin{array}{l}\text { Trigo- } \\
\text { nelline }\end{array}$ & $\begin{array}{l}\text { L-Arabi- } \\
\text { nose }\end{array}$ & & \\
\hline Olive & 58 & 100 & 12 & 0 & 50 & 0 & 0 & 0 & 45 & 50 & 37 & 13 & 0 \\
\hline Ash & 39 & 21 & 82 & 53 & 92 & 23 & 13 & 18 & 92 & 0 & 95 & 100 & 21 \\
\hline Oleander & 33 & 100 & 18 & 0 & 76 & 0 & 0 & 0 & 3 & 0 & 79 & 97 & 0 \\
\hline Jasmine & 3 & 100 & 66 & 0 & 100 & 0 & 0 & 0 & 0 & 0 & 100 & 100 & 0 \\
\hline Phillyrea sp. & 4 & 100 & 0 & 0 & 100 & 0 & 0 & 0 & 0 & 0 & 100 & 100 & 0 \\
\hline Privet & 6 & 100 & 0 & 0 & 16 & 0 & 0 & 0 & 0 & 0 & 100 & 16 & 16 \\
\hline
\end{tabular}


TABLE 6. Levels of DNA relatedness among Pseudomonas strains

\begin{tabular}{|c|c|c|c|}
\hline \multicolumn{2}{|c|}{ Unlabeled DNA from: } & \multicolumn{2}{|c|}{$\begin{array}{l}\% \text { of relative binding at } 60^{\circ} \mathrm{C} \text { with } \\
\text { labeled DNA from: }\end{array}$} \\
\hline Taxon & Strain (host) & $\begin{array}{l}\text { P. syringae } \\
\text { CFBP } 1392^{\mathrm{T}}\end{array}$ & $\begin{array}{l}\text { P. savastanoi } \\
\text { CFBP } 1670^{\mathrm{T}}\end{array}$ \\
\hline \multirow{23}{*}{ P. savastanoi pv. savastanoi (phenon VI) } & CFBP $1670^{\mathrm{T}}$ (olive) & 54 & 100 \\
\hline & K124-4 (olive) & 53 & 82 \\
\hline & T35-1 (olive) & 49 & 87 \\
\hline & T12-6 (olive) & 64 & 83 \\
\hline & K23-15 (olive) & 49 & 93 \\
\hline & CFBP 2088 (oleander) & 58 & $79(3)^{a}$ \\
\hline & L145-2 (oleander) & 52 & 88 \\
\hline & T37-6 (oleander) & 49 & 90 \\
\hline & L36-7 (oleander) & 50 & 84 \\
\hline & L86-1 (oleander) & 53 & 81 \\
\hline & CFBP 1838 (ash) & 58 & 86 \\
\hline & CFBP 2093 (ash) & 58 & 97 \\
\hline & T36-3 (ash) & 55 & 92 \\
\hline & T5-1 (ash) & 47 & $75(1.5)$ \\
\hline & T12-7 (jasmine) & 60 & 92 \\
\hline & CFBP 1751 (jasmine) & 53 & 88 \\
\hline & $\mathrm{T} 12-10$ (jasmine) & 54 & 83 \\
\hline & Phi (Phillyrea sp.) & 54 & 100 \\
\hline & T51-3 (Phillyrea sp.) & 62 & 93 \\
\hline & T51-1 (Phillyrea sp.) & 50 & 81 \\
\hline & T37-11 (privet) & 59 & 86 \\
\hline & T35-10 (privet) & 57 & 86 \\
\hline & T37-15 (privet) & 53 & 90 \\
\hline P. savastanoi pv. phaseolicola (phenon VII) & CFBP $1390^{\mathrm{T}}$ & 56 & 83 \\
\hline P. savastanoi pv. glycinea (phenon VII) & CFBP $2214^{\mathrm{T}}$ & 50 & $72(2.5)$ \\
\hline$P$. syringae pv. syringae (isolated phenotype) & CFBP $1392^{\mathrm{T}}$ & 100 & 43 \\
\hline$P$. syringae pv. persicae (phenon IVb) & CFBP $1573^{T}$ & 46 & 41 \\
\hline$P$. syringae pv. tomato (phenon IVd) & CFBP $2212^{\mathrm{T}}$ & 51 & 47 \\
\hline$P$. viridiflava (isolated phenotype) & CFBP $2107^{\mathrm{T}}$ & 49 & 44 \\
\hline P. cichorii (isolated phenotype) & CFBP $2101^{T}$ & 25 & 24 \\
\hline$P$. marginalis (phenon I) & CFBP $1387^{T}$ & 21 & 22 \\
\hline P. fluorescens (phenon II) & CFBP $2102^{\mathrm{T}}$ & 20 & 21 \\
\hline P. putida (phenon I) & CFBP $2066^{T}$ & 9 & 10 \\
\hline
\end{tabular}

${ }^{a}$ The values in parentheses are $\Delta T_{m}$ values (in degrees Celsius).

Pseudomonas savastanoi and that this species should include three pathovars, $P$. savastanoi pv. savastanoi, $P$. savastanoi pv. glycinea, and $P$. savastanoi pv. phaseolicola.

Description of Pseudomonas savastanoi (Smith). Pseudomonas savastanoi (sa.vas.ta'no.i. L.gen.n. savastanoi of Savastano, the first worker who studied olive knot disease). Gram-negative rods that are 0.4 to 0.8 by 1.0 to $3.0 \mu \mathrm{m}$ and motile by means of one to four polar flagella. Rather slow growing. Colonies are white or cream, smooth, flat, and glistening with entire or erose margins on YBGA. Produces a hypersensitive reaction on tobacco leaves. Metabolism is respiratory. Oxidase negative. Nitrates are not reduced. Blue fluorescent pigment is produced under UV light on King B medium. Arginine test (Thornley) negative. Esculin, gelatin, and starch are not hydrolyzed. Assimilates sucrose, L-arabinose, gluconate, caprylate, fumarate, DL-glycerate, L-malate, pyruvate, citrate, $\mathrm{D}-\alpha$-alanine, and L-proline. Does not assimilate lactose, L-xylose, adonitol, 2-aminobutyrate, DL-lactate, DL-3-hydroxybutyrate, D-(-)-tartrate, L-cysteine, L-methionine, and L-valine. Strains isolated from members of various genera of the Oleaceae and $N$. oleander do not produce levan, whereas strains isolated from $F$. excelsior
L. (21\%), Phaseolus vulgaris, and Glycine max L. do produce levan. The $\mathrm{G}+\mathrm{C}$ content of the DNA is $60 \mathrm{~mol} \%$ (4).

$P$. savastanoi pv. savastanoi causes knots, galls, and cankers on members of the various genera of the Oleaceae and $N$. oleander L.; $P$. savastanoi pv. glycinea causes bacterial blight of soybean; and $P$. savastano $\mathrm{pv}$. phaseolicola causes halo blight of bean.

The type strain is strain NCPPB 639 (= ATCC $13522=$ ICMP 4352 = CFBP 1670).

Description of the type strain. Strain NCPPB $639^{\mathrm{T}}$ was isolated from $O$. europea L. in Yugoslavia. This strain has physiological and biochemical characteristics that are typical of the species. In addition, it does not assimilate sorbitol and trans-aconitate.

\section{REFERENCES}

1. Brenner, D. J., A. C. McWorter, J. K. Leete Knutson, and A. G. Steigerwalt. 1982. Escherichia vulneris: a new species of Enterobacteriaceae associated with human wounds. J. Clin. Microbiol. 15:1133-1140.

2. Brown, N. A. 1932. Canker of ash trees produced by a variety of the olive tubercule organism, Bacterium savastanoi. J. Agric. Res. 44:701-722. 
3. Crosa, J. M., D. J. Brenner, and S. Falkow. 1973. Use of a single-strand-specific nuclease for analysis of bacterial and plasmid deoxyribonucleic acid homo- and hetroduplexes. J. Bacteriol. 115:904-911.

4. De Ley, J. 1968. DNA base composition and hybridization in the taxonomy of phytopathogenic bacteria. Annu. Rev. Phytopathol. 6:63-90.

5. Dye, D. W., J. F. Bradbury, M. Goto, A. C. Hayward, R. A. Lelliott, and M. N. Schroth. 1980. International standards for naming pathovars of phytopathogenic bacteria and a list of pathovar names and pathotype strains. Rev. Plant Pathol. 59:153-168.

6. Ferraris, T. 1926. Trattato di patologia e terapia vegetale, vol. 1, 3rd ed. Hopli, Milan, Italy.

6a.Gardan, L. Unpublished data.

7. Gardan, L., S. Cottin, C. Bollet, and G. Hunault. 1991. Phenotypic heterogeneity of Pseudomonas syringae. Res. Microbiol. 142:995-1003.

8. Grimont, P. A. D., M. Y. Popoff, F. Grimont, C. Coynautt, and M. Lemelin. 1980. Reproductibility and correlation study of three deoxyribonucleic acid hybridization procedures. Curr. Microbiol. 4:325-330.

9. Hildebrand, D. C. 1971. Pectate and pectin gels for differentiation of Pseudomonas sp. and other bacterial plant pathogens. Phytopathology 61:1430-1436.

10. Hildebrand, D. C., and M. N. Schroth. 1972. Identification of the fluorescent pseudomonads, p. 281-287. In H. P. Maas Geesteranus (ed.), Proceedings of the Third International Conference on Plant Pathogenic Bacteria. Pudoc, Wageningen, The Netherlands.

11. Janse, J. D. 1981. The bacterial disease of ash (Fraxinus excelsior) caused by Pseudomonas syringae subsp. savastanoi pv. fraxini. II. Etiology and taxonomic considerations. Eur. J. For. Pathol. 11:425-438.

12. Janse, J. D. 1982. Pseudomonas syringae subsp. savastanoi (ex Smith) subsp. nov., nom. rev., the bacterium causing excrescences on Oleaceae and Nerium oleander L. Int. J. Syst. Bacteriol. 32:166-169.

13. Lapage, S. P., S. Bascomb, W. R. Wilcox, and M. A. Curtis. 1973. Identification of bacteria by computer: general aspects and perspectives. J. Gen. Microbiol. 77:273-290.

14. Lapage, S. P., P. H. A. Sneath, E. F. Lessel, V. B. D. Skerman, H. P. R. Seeliger, and W. A. Clark (ed.). 1975. International code of nomenclature of bacteria. 1975 Revision. American Society for Microbiology, Washington, D.C.

15. Lelliott, R. A., E. Billing, and A. C. Hayward. 1966. A determinative scheme for the fluorescent plant pathogenic pseudomonads. J. Appl. Bacteriol. 29:470-489.

16. Misaghi, I., and R. G. Grogan. 1969. Nutritional and biochemical comparisons of plant pathogenic and saprophytic fluorescent pseudomonads. Phytopathology 59:1436-1451.

17. Moore, W. E. C., and L. V. H. Moore (ed.). 1989. Index of bacterial and yeast nomenclatural changes, p. 49. American Society for Microbiology, Washington, D.C.

18. Pecnold, P. C., and R. G. Grogan. 1973. Deoxyribonucleic acid homology groups among phytopathogenic Pseudomonas species. Int. J. Syst. Bacteriol. 23:111-121.

19. Prunier, J. P., and P. Kaiser. 1964. Etude de l'activité pectinolytique chez des bactéries phytopathogènes et saprophytes des plantes. I. Recherche des enzymes pectinolytiques. Ann. Epiphyt. (Paris) 15:205-219.

20. Sands, D. C., M. N. Schroth, and D. C. Hildebrand. 1970. Taxonomy of phytopathogenic pseudomonads. J. Bacteriol. 101:9-23.

21. Skerman, V. B. D., V. McGowan, and P. H. A. Sneath (ed.). 1989. Approved lists of bacterial names. Amended edition. American Society for Microbiology, Washington, D.C.

22. Smith, E. D. 1908. Recent studies on the olive tubercule organism. Bull. Bur. Plant Ind. U. S. Dep. Agric. 131:25-43.

23. Sneath, P. H. A., and R. R. Sokal. 1973. Numerical taxonomy. The principles and practice of numerical classification. W. $H$. Freeman, San Francisco.

24. Stevens, F. L. 1913. The fungi which cause plant disease. Macmillan Co., New York.

25. Wilson, E. E., M. G. Heskett, M. L. Johnson, and T. Kosuge. 1972. Metabolic behaviour of Pseudomonas savastanoi isolates from olive and oleander on certain carbohydrates and amino substrates. Phytopathology 62:350-355.

26. Young, J. M., J. F. Bradbury, R. E. Davis, R. S. Dickey, and G. L. Ercolani. 1991. Nomenclatural revisions of plant pathogenic bacteria and list of names, 1980-1988. Rev. Plant Pathol. 70:221.

27. Young, J. M., D. W. Dye, J. F. Bradbury, C. G. Panagopoulos, and C. F. Robb. 1978. A proposed nomenclature and classification for plant pathogenic bacteria. N. Z. J. Agric. Res. 21:153177. 